\title{
The New Bivariate Conway-Maxwell-Poisson Distribution Obtained by the Crossing Method
}

\author{
Rufin Bidounga ${ }^{1}$, Evrand Giles Brunel Mandangui Maloumbi ${ }^{2}$, Réolie Foxie Mizélé Kitoti ${ }^{2}$ and Dominique Mizère ${ }^{2}$ \\ ${ }^{1}$ Normal School Higher, Marien Ngouabi University, BP 69, Brazzaville, Congo \\ ${ }^{2}$ Faculty of sciences and technics, Marien Ngouabi University, BP 69, Brazzaville, Congo \\ ${ }^{1,2}$ Laboratory of statistics and analysis of data(Labsad), faculty of sciences and technics, Marien Ngouabi University, BP \\ 69, Brazzaville, Congo
}

Correspondence: Evrand Giles Brunel Mandangui Maloumbi, Faculty of sciences and technics, Marien Ngouabi University, BP 69, Brazzaville, Congo

Received: July 30, 2020 Accepted: August 31, 2020 Online Published: September 13, 2020

doi:10.5539/ijsp.v9n6p1 URL: https://doi.org/10.5539/ijsp.v9n6p1

\begin{abstract}
Kimberly et al. had proposed in 2016 a bivariate function as a bivariate Conway-Maxwell-Poisson distribution (COMPoisson) using the generalized bivariate Poisson distribution and the probability generating functions of the follow distributions: bivariate bernoulli, bivariate Poisson, bivariate geometric and bivariate binomial. By examining this paper we have shown that this bivariate function is constant and it double series is divergent, when it should have been 1 . To overcome this deadlock, we propose a new bivariate Conway-Maxwell-Poisson distribution which is definetely a probability distribution based on the crossing method, method highlighted by Elion et al. in 2016 and revisited by Batsindila et al. and Mandangui et al. in 2019. And this is the purpose of this paper. To this bivariate distribution is attached two generalized linear models (GLM) whose resolution allows us to highlight, not only the independence between the variables forming the couple, but also the effect of the factors (or predictors) on these variables. The resulting correlation is negative, zero or positive depending on the values of a parameter; in particular for the bivariate Poisson distribution according to Berkhout and Plug. A simulation of data will be given at the end of the article to illustrate the model.
\end{abstract}

Keywords: univariate Conway-Maxwell-Poisson distribution, bivariate Conway-Maxwell-Poisson distribution, generalized linear models, maxLik

AMS 2010 Mathematics subject classification: 62F10; 62J12

\section{Introduction}

The COM-Poisson distribution was introduced by Conway and Maxwell in 1962 and revisited by Shmueli et al. in 2005. It has a mass function

$$
P(X=x / \lambda, v)=\frac{\lambda^{x}}{(x !)^{v}} \frac{1}{Z(\lambda, v)}, \quad x \in \mathbb{N}, \lambda>0, v \geq 0,
$$

with

$$
Z(\lambda, v)=\sum_{n=0}^{+\infty} \frac{\lambda^{n}}{(n !)^{v}}
$$

It has a probability generating function $g_{X}(Z)=\frac{Z(\lambda z, v)}{Z(\lambda, v)}$, and for moment generating function $M_{X}(S)=\frac{Z\left(\lambda e^{s}, v\right)}{Z(\lambda, v)}$.

\subsection{Properties}

The COM-Poisson distribution is a generalization of some usual distributions such as:

1. when $v=1$, then $P(X=x / \lambda, v)$ is Poisson distribution of the parameter $\lambda$,

2. when $v=0$ and $0<\lambda<1$, then $P(X=x / \lambda, v)=\lambda^{x}(1-\lambda)$ is a geometric distribution,

3. when $v \rightarrow+\infty$, then $P(X=x / \lambda, v)$ converge to the Bernoulli distribution of parameter $\frac{\lambda}{1+\lambda}$.

\subsection{The COM-Poisson Distribution Is a Weighted Poisson Distribution}

The COM-Poisson distribution is a weighted Poisson distribution of weight function(Kokonendji et al.,2008) $w(x, v)=$ $(x !)^{1-v}$ and constant of normalization $E_{\lambda}[w(Y)]=e^{-\lambda} Z(\lambda, v)$. 


\subsection{Characteristics}

The COM-Poisson distribution has characteristics

$$
E_{\lambda}(X)=\frac{\partial}{\partial \ln \lambda} \ln [Z(\lambda, v)]
$$

and

$$
\operatorname{Var}(X)=\frac{\partial E_{\lambda}(X)}{\partial \ln \lambda}
$$

Indeed, $X$ follow the weighted Poisson distribution variable, its characteristics are equal to (Kokonendji et al.,2008)

$$
\begin{aligned}
E_{\lambda}(X) & =\lambda\left(1+\frac{\partial}{\partial \lambda} \ln \left[E_{\lambda}(w(Y, v))\right]\right), \\
& =\lambda \frac{\partial}{\partial \lambda} \ln [Z(\lambda, v)] \\
& =\frac{\partial}{\partial \ln \lambda} \ln [Z(\lambda, v)] .
\end{aligned}
$$

Because $\frac{\partial}{\partial \lambda}=\frac{\partial \ln \lambda}{\partial \lambda} \frac{\partial}{\partial \ln \lambda}=\frac{1}{\lambda} \frac{\partial}{\partial \ln \lambda}$ and $\lambda \frac{\partial}{\partial \lambda}=\frac{\partial}{\partial \ln \lambda}$.

$$
\begin{aligned}
\operatorname{Var}(X) & =E_{\lambda}(X)+\lambda^{2} \frac{\partial^{2}}{\partial \lambda^{2}} \ln \left[E_{\lambda}(w(Y, v))\right], \\
& =E_{\lambda}(X)+\lambda^{2} \frac{\partial^{2}}{\partial \lambda^{2}}(-\lambda+\ln [Z(\lambda, v)]), \\
& =E_{\lambda}(X)+\lambda^{2} \frac{\partial}{\partial \lambda}\left[-1+\frac{\partial}{\partial \lambda} \ln [Z(\lambda, v)],\right. \\
& =E_{\lambda}(X)+\lambda^{2} \frac{\partial}{\partial \lambda}\left[\frac{1}{\lambda} E_{\lambda}(X)\right], \\
& =E_{\lambda}(X)+\lambda \frac{\partial}{\partial \lambda}\left[E_{\lambda}(X)\right]-E_{\lambda}(X), \\
& =\frac{\partial E_{\lambda}(X)}{\partial \ln \lambda} .
\end{aligned}
$$

\subsection{Overdispersion and Underdispersion}

The COM-Poisson variable is(Kokonendji et al.,2008) overdispersed when $v \in[0,1[$ and is underdispersed, when $v>1$. 1.5 Approximation of $Z(\lambda, v)$

We have the following approximations (Shmueli et al,2005)

1- When there is an integer $N$ such as for $n>N, \frac{\lambda}{n^{v}}<1$, then $Z(\lambda, v) \simeq \sum_{y=0}^{n} \frac{\lambda^{y}}{(y !)^{v}}$,

2- When $\lambda$ is higher, then $Z(\lambda, v)=\frac{\exp \left(\nu \lambda \frac{1}{v}\right)}{\lambda^{\frac{(\nu-1)}{2 v}}(2 \pi)^{\frac{(\nu-1)}{2}} \sqrt{v}}\left\{1+0\left(\lambda^{-\frac{1}{v}}\right)\right\}$.

In the section (2), we will present the bivariate COM-Poisson distribution according to Kimberly et al.(2016) and in the section (3), the new bivariate COM-Poisson distribution. Finally, in the section (4), a simulation of the data will be carried out to illustrate the model.

\section{The Bivariate COM-Poisson Distribution According to Kimberly et al. (2016)}

In this section, we will present only a few salient points of this study and for details see the article by Kimberly et al.(2016). Let $(0,0),(0,1),(1,0)$ and $(1,1)$ the values of a bivariate Bernoulli variable of probabilities $p_{00}, p_{01}, p_{10}$ and $p_{11}$ with $p_{00}+p_{01}+p_{10}+p_{11}=1$.

Let following the multinomial expressions (Johnson et al., 1997; Kimberly et al.,2016)

$$
\left(\begin{array}{cccc} 
& a & & \\
b_{1} & b_{2} & \cdots & b_{k}
\end{array}\right)=\frac{a !}{\prod_{i=1}^{k} b_{i} !} \text {, with } \sum_{i=1}^{k} b_{i}=a, b_{i} \in \mathbb{N}(i=1, k),
$$


and

$$
\begin{gathered}
\left(A+B t_{1}+C t_{2}+F t_{1} t_{2}\right)^{n}=\sum_{a, b, c, d=0 ; a+b+c+d \leq n}^{n}\left(\begin{array}{cccc}
n & & \\
a & b & c & d
\end{array}\right) A^{a}\left(B t_{1}\right)^{b}\left(C t_{2}\right)^{c}\left(F t_{1} t_{2}\right)^{d}, \\
=\sum_{a, b, c, d=0 ; a+b+c+d \leq n}^{n}\left(\begin{array}{cccc}
n & & \\
a & b & c & d
\end{array}\right) A^{a} B^{b} C^{c} F^{n-a-b-c} t_{1}^{n-a-b-c} t_{2}^{n-a-b},
\end{gathered}
$$

We have the following result

\subsection{Proposition}

By posing $t_{1}=t_{2}=1, A=\lambda p_{00} ; B=\lambda p_{10} ; C=\lambda p_{01}$ and $F=\lambda p_{11}$, with $\lambda>0$, we have the identity

$$
\sum_{a=n-x-y}^{n}\left(\begin{array}{c}
n \\
a, \quad n-a-y, \quad n-a-x, \quad x+y+a-n
\end{array}\right) p_{00}^{a} p_{10}^{n-a-y} p_{01}^{n-a-x} p_{11}^{x+y-n+a} \equiv 1 .
$$

Indeed,

the left side of the expression (4) noted $G$ is equal to

$$
G=\lambda^{n}\left(p_{00}+p_{01}+p_{10}+p_{11}\right)^{n}=\lambda^{n} .
$$

And the right part denoted $D$ is equal to

$$
\begin{aligned}
D & =\sum_{a, b, c, d=0 ; a+b+c+d \leq n}^{n}\left(\begin{array}{llll} 
& n & & \\
a & b & c & d
\end{array}\right) A^{a}(B)^{b}(C)^{c}(F)^{n-a-b-c}, \\
& =\lambda^{n} \sum_{a, b, c, d=0 ; a+b+c+d \leq n}^{n}\left(\begin{array}{llll} 
& n & \\
a & b & c & d
\end{array}\right) p_{00}^{a} p_{10}^{b} p_{01}^{c} p_{11}^{n-a-b-c} .
\end{aligned}
$$

Given that $G=D$, we have the following result

$$
\sum_{a, b, c, d=0 ; a+b+c+d \leq n}^{n}\left(\begin{array}{cccc} 
& n & & \\
a & b & c & d
\end{array}\right) p_{00}^{a} p_{10}^{b} p_{01}^{c} p_{11}^{n-a-b-c} \equiv 1 .
$$

By posing

$x=n-a-c$ and $y=n-a-b$, we have:

$b=n-a-y, c=n-a-x$ and $n-a-b-c=x+y+a-n$

the final result is given by

$$
\sum_{a=n-x-y}^{n}\left(\begin{array}{c}
n \\
a, \quad n-a-y, \quad n-a-x, \quad x+y+a-n
\end{array}\right) p_{00}^{a} p_{10}^{n-a-y} p_{01}^{n-a-x} p_{11}^{x+y-n+a} \equiv 1 .
$$

\subsection{Corollary}

The following mass function proposed by Kimberly et al.(2016)

$$
\begin{aligned}
& \operatorname{Pr}(Y=y, X=x)=\frac{1}{Z(\lambda, v)} \sum_{n=0}^{\infty} \frac{\lambda^{n}}{(n !)^{v}} \times \\
& \times \sum_{a=n-x-y}^{n}\left(\begin{array}{c}
n \\
a, \quad n-a-y, \quad n-a-x, \quad x+y+a-n
\end{array}\right) p_{00}^{a} p_{10}^{n-a-y} p_{01}^{n-a-x} p_{11}^{x+y-n+a},
\end{aligned}
$$

as a bivariate COM-Poisson distribution is not a probability.

Indeed, we have

$$
\operatorname{Pr}(Y=y, X=x)=\frac{1}{Z(\lambda, v)} \sum_{n=0}^{\infty} \frac{\lambda^{n}}{(n !)^{v}}=1,
$$

and it double series $\sum_{x, y=0}^{+\infty} \operatorname{Pr}(Y=y, X=x)$ is divergent. This bivariate function is therefore not a probability because this double series should have been 1 . 


\section{The New Bivariate COM-Poisson Distribution}

\subsection{Definition: The Crossing Method}

Let us consider two random variables $X$ and $Y$ which follow univariate COM-Poisson distribution of mass functions

$$
P\left(X=x / \lambda_{1}, v_{1}\right)=\frac{\lambda_{1}^{x}}{(x !)^{v_{1}}} \frac{1}{Z\left(\lambda_{1}, v_{1}\right)}, \quad x \in \mathbb{N}, \nu_{1} \in \mathbb{R}_{+}, \lambda_{1} \in \mathbb{R}_{+}^{*},
$$

and

$$
P\left(Y=y / \lambda_{2}, v_{2}\right)=\frac{\lambda_{2}^{y}}{(y !)^{v_{2}}} \frac{1}{Z\left(\lambda_{2}, \nu_{2}\right)}, \quad y \in \mathbb{N}, v_{2} \in \mathbb{R}_{+}, \lambda_{2} \in \mathbb{R}_{+}^{*} .
$$

The couple $(X, Y)$ follows the bivariate COM-Poisson distribution if and only if it mass function is equal to (Elion et al., 2016; Batsindila et al., 2019 and Mandangui et al.,2019)

$$
\begin{array}{r}
P\left(X=x, Y=y / \lambda_{1}, v_{1}, \lambda_{2}, v_{2}\right)=\frac{\lambda_{1}^{x}}{(x !)^{v_{1}}} \frac{\lambda_{2}^{y}}{(y !)^{v_{2}}} \frac{1}{Z\left(\lambda_{1}, v_{1}\right)} \frac{1}{Z\left(\lambda_{2}, v_{2}\right)}, \\
x, y \in \mathbb{N}, v_{1}, v_{2} \in \mathbb{R}_{+}, \lambda_{1}, \lambda_{2} \in \mathbb{R}_{+}^{*},
\end{array}
$$

under the conditions

$$
\ln \lambda_{1}=t^{\prime} \beta_{1}
$$

and

$$
\ln \lambda_{2}=t^{\prime} \beta_{2}+\eta x
$$

$X$ is the response variable of the model (7) and $Y$ that of the model (8), with $t^{\prime}=\left(t_{1}, t_{2}, \ldots, t_{p}\right)$ the vector of predictors variables or factors. The expression (8) leads to the fact that $P\left(Y=y / \lambda_{2}, v_{2}\right)=P(Y=y / X=x)$ is a conditional probability.

One has the following relation

$$
P\left(X=x, Y=y / \lambda_{1}, v_{1}, \lambda_{2}, v_{2}\right)=P\left(X=x / \lambda_{1}, v_{1}\right) \times P(Y=y / X=x)
$$

$P\left(X=x / \lambda_{1}, v_{1}\right)$ is the marginal distribution. This bivariate distribution is definetely a probability distribution.

When $\eta=0$, the variables $X$ and $Y$ are independent.

When $v_{1}=v_{2}=1$, the bivariate COM-Poisson distribution is identical to the bivariate Poisson distribution according to Berkout and Plug(2004).

\subsection{Characteristics}

A simple application of proposition 1 from the work of Batsindila et al.(2019), gives us the following results

$$
\begin{gathered}
E_{\lambda_{2}}(Y)=e^{t^{\prime} \beta_{2}+\lambda_{1}\left(e^{\eta}-1\right)} \frac{Z\left(\lambda_{1} e^{\eta}, v_{1}\right)}{Z\left(\lambda_{1}, v_{1}\right)} \\
\operatorname{Var}(Y)=\left(e^{2 \lambda_{1}\left(e^{\eta}-1\right)} \frac{Z\left(\lambda_{1}, v_{1}\right)}{Z\left(\lambda_{1} e^{\eta}, v_{1}\right)}-1\right) \\
\operatorname{Cov}(X, Y)=E_{\lambda_{1}}(X) E_{\lambda_{2}}(Y)\left(e^{\eta}-1\right)
\end{gathered}
$$

The expressions (8) and (11) confirm that the variables $X$ and $Y$ are independent if and only if the parameter $\eta=0$. The resulting correlation is negative, zero or positive depending on whether the value of the parameter $\eta$ is negative, zero or positive in particular for the bivariate Poisson distribution according to Berkhout and Plug(2004).

Remarque 1. The variable X follow the COM-Poisson distribution, its characteristics have been given in the subsection 1.3. 


\subsection{Ratio of Successives Probabilities and Estimation of Parameters $\lambda_{1}, v_{1}, \lambda_{2}, v_{2}$}

Let

$$
p_{x-1}=P\left(X=x-1 / \lambda_{1}, v_{1}\right)=\frac{\lambda_{1}^{x-1}}{((x-1) !)^{v_{1}}} \frac{1}{Z\left(\lambda_{1}, v_{1}\right)}, \quad x \in \mathbb{N}^{*}, v_{1} \in \mathbb{R}_{+}, \lambda_{1} \in \mathbb{R}_{+}^{*} .
$$

The ratio of successives probabilities of the COM-Poisson distribution is equal to (Shmueli et al.,2005)

$\frac{p_{x-1}}{p_{x}}=\frac{x^{\nu_{1}}}{\lambda_{1}}$, which has logorithm

$$
\ln \left[\frac{p_{x-1}}{p_{x}}\right]=-\ln \lambda_{1}+v_{1} \ln x
$$

we have by symmetry,

$$
\ln \left[\frac{p_{y-1}}{p_{y}}\right]=-\ln \lambda_{2}+v_{2} \ln y
$$

When the plot of the set data $\left\{\left(\ln x, \ln \left[\frac{p_{x-1}}{p_{x}}\right]\right) / x>1\right\}$ is adjusted by a straight line, then the random variable $X$ follow a COM-Poisson distribution(Shmueli et al.,2005). Thanks to the distribution of large numbers, successive probabilities $p_{x}\left(p_{y}\right)$ can be replaced by successives frequencies $f_{x}\left(f_{y}\right)$ associated with $x(y)$. In this case, the expression $\ln \left[\frac{f_{x-1}}{f_{x}}\right]$ which is called $\log$-ratio successives frequencies, will be used as a replacement for $\ln \left[\frac{p_{x-1}}{p_{x}}\right]$, the response variable with $\ln x$ the explanatory variable in the model (12). Although the basic assumtions of homoscedasticity and independence of theses log-ratio are not established(Cf. Shmueli et al.,2005), the regression lines of (12) and (13) allow to calculate the estimators of $\lambda_{1}, v_{1}, \lambda_{2}, v_{2}$ noted respectively $\widehat{\lambda_{1}}, \widehat{v_{1}}, \widehat{\lambda_{2}}, \widehat{v_{2}}$.

\subsection{The Log Likelihood Estimation of Parameter $\beta_{1}, \beta_{2}$ et $\eta$}

Let consider the distributions $P\left(X=x / \lambda_{1}, \widehat{v_{1}}\right), P\left(Y=y / \lambda_{2}, \widehat{v_{2}}\right)$ and generalized linear models (7) and (8), we will calculate the maximum likelihood estimators of the parameters $\beta_{1}, \beta_{2}$ and $\eta$ in order to be able to highlight, not only the independence between the variables $X$ and $Y$ but also the effect of factors on these variables.

\subsubsection{The Log Likelihood Function}

The log likelihood function of the bivariate COM-Poisson distribution is equal to

$$
\ln P=x \ln \lambda_{1}+y \ln \lambda_{2}-\widehat{v_{1}} \ln (x !)-\widehat{v_{2}} \ln (y !)-\ln \left[\sum_{x=0}^{n} \frac{\lambda_{1}^{x}}{(x !)^{\widehat{v_{1}}}}\right]-\ln \left[\sum_{y=0}^{n} \frac{\lambda_{2}^{y}}{(y !)^{\widehat{v_{2}}}}\right] .
$$

By the replacement in the expressions (7) and (8) we have:

$$
\begin{aligned}
\ln P & =x t^{\prime} \beta_{1}+y\left(t^{\prime} \beta_{2}+\eta x\right)-\widehat{v_{1}} \ln (x !)-\widehat{v_{2}} \ln (y !)- \\
& -\ln \left[\sum_{x=0}^{n} \frac{\left(e^{t^{\prime} \beta_{1}}\right)^{x}}{(x !)^{\widehat{v_{1}}}}\right]-\ln \left[\sum_{y=0}^{n} \frac{\left(e^{t^{\prime} \beta_{2}+\eta x}\right)^{y}}{(y !)^{\widehat{v_{2}}}}\right]
\end{aligned}
$$

after development we find

$$
\ln P=x t^{\prime} \beta_{1}+y t^{\prime} \beta_{2}+y \eta x-\widehat{v_{1}} \ln (x !)-\widehat{v_{2}} \ln (y !)-\ln \left[\sum_{x=0}^{n} \frac{e^{t^{\prime} \beta_{1} x}}{(x !)^{\widehat{v_{1}}}}\right]-\ln \left[\sum_{y=0}^{n} \frac{e^{t^{\prime} \beta_{2} y+\eta x y}}{(y !)^{\widehat{v_{2}}}}\right]
$$

\subsubsection{Parameters Estimate $\beta_{1}, \beta_{2}$ et $\eta$}

The parameters $\beta_{1}, \beta_{2}$ and $\eta$ will be estimated by the maximum likelihood method. Either a sample $\left(x_{i}, y_{i}\right)$ of size $n$ values of the couple of variables $(X, Y)$ of probability density $P$.

The likelihood of observing this sample is equal to

$$
\mathcal{L}=\prod_{i=1}^{n} P\left(x_{i}, y_{i}, \lambda_{1}, \widehat{v_{1}}, \lambda_{2}, \widehat{v_{2}}\right)
$$


By applying the logarithm, we have:

$$
\begin{aligned}
L=\ln \mathcal{L} & =\ln \left[\prod_{i=1}^{n} P\left(x_{i}, y_{i}, \lambda_{1}, \widehat{v_{1}}, \lambda_{2}, \widehat{v_{2}}\right)\right]=\sum_{i=1}^{n} \ln P\left(x_{i}, y_{i}, \lambda_{1}, \widehat{v_{1}}, \lambda_{2}, \widehat{v_{2}}\right) \\
& =\sum_{i=1}^{n} \ln \left[P\left(x_{i} ; \lambda_{1}, \widehat{v_{1}}\right) \times P\left(y_{i} ; \lambda_{2}, \widehat{v_{2}}\right)\right]=\sum_{i=1}^{n} \ln P\left(x_{i} ; \lambda_{1}, \widehat{v_{1}}\right)+\sum_{i=1}^{n} \ln P\left(y_{i} ; \lambda_{2}, \widehat{v_{2}}\right)
\end{aligned}
$$

where the function $P$ is defined by the expression (6). By using the expressions (7) and (8) one has

$$
L=\sum_{i=1}^{n} \ln P\left(x_{i} ; \beta_{1}, \widehat{v_{1}}\right)+\sum_{i=1}^{n} \ln P\left(y_{i} ; \beta_{2}, \eta, \widehat{v_{2}}\right)
$$

We will use the function maxLik of the statistical computer software $\mathrm{R}$ to determine these estimators.

\section{Illustration}

\subsection{Data and Software Used}

To illustrate this method, we will simulate the COM-Poisson data using the Statistics computer software R data processing software. Let us recall that the COM-Poisson distribution with two parameters, one canonical and the other dispersion.

The COM-Poisson data of the random variables $X$ and $Y$ simulated of size $N=50$ are given in the table (1) and (2)

Table 1. Variable $X$ for $\lambda_{1}=2$ and $v_{1}=2$

\begin{tabular}{cccccccc}
\hline$X$ & 0 & 1 & 2 & 3 & $\lambda_{1}$ & $v_{1}$ & $N$ \\
\hline Obs. & 7 & 32 & 8 & 3 & 2 & 2 & 50 \\
\hline
\end{tabular}

Table 2. Variable $Y$ for $\lambda_{2}=3$ and $\nu_{2}=2$

\begin{tabular}{ccccccccc}
\hline$Y$ & 0 & 1 & 2 & 3 & 4 & $\lambda_{2}$ & $v_{2}$ & $N$ \\
\hline Obs. & 8 & 20 & 16 & 5 & 1 & 3 & 2 & 50 \\
\hline
\end{tabular}

Table 3. Simulated data of the Poisson variable t of parameter $\lambda=2$

\begin{tabular}{ccccccccc}
\hline$t$ & 0 & 1 & 2 & 3 & 4 & 5 & 7 & $\mathrm{~N}$ \\
\hline Obs. & 10 & 11 & 14 & 11 & 2 & 1 & 1 & 50 \\
\hline
\end{tabular}

Table 4. Elementary statistics

\begin{tabular}{cccc}
\hline Variables & Mean & Variance & Indice of Fisher \\
\hline$X$ & 1.14 & 0.5310204 & 0.4658074 \\
$Y$ & 1.42 & 0.9016327 & 0.6349526 \\
\hline
\end{tabular}

From the table (4), the simulated data is underdispersed because the Fisher Indices are small than 1. 


\subsubsection{Parameters Estimate $\lambda_{1}, v_{1}, \lambda_{2}, v_{2}$}

Table 5. Regression coefficients of model (12)

\begin{tabular}{ccccc}
\hline \hline Variables & Parameters & $S_{\hat{\beta}}$ & $k_{j}=\frac{\hat{\beta}}{S_{\hat{\beta}}}$ & $P\left(>\left|k_{j}\right|\right)$ \\
\hline Intercept & 0.7568 & 1.7238 & 0.439 & 0.737 \\
$\ln x$ & 2.5757 & 2.2984 & 1.121 & 0.464 \\
\hline \hline
\end{tabular}

Table 6. Regression coefficients of model (13)

\begin{tabular}{ccccc}
\hline \hline Variables & Parameters & $S_{\hat{\beta}}$ & $k_{j}=\frac{\hat{\beta}}{S_{\hat{\beta}}}$ & $P\left(>\left|k_{j}\right|\right)$ \\
\hline Intercept & -0.09317 & 0.78709 & -0.118 & 0.9166 \\
lny & 3.21664 & 0.82861 & 3.882 & 0.0604 \\
\hline \hline
\end{tabular}

Hence the estimated parameters of the models (12) and (13) are (table (7))

Table 7. Estimated parameters

\begin{tabular}{ccc}
\hline \hline Variables & \multicolumn{2}{c}{ Parameters } \\
\hline \hline$X$ & $\widehat{\lambda_{1}}=0.4691654$ & $\widehat{v_{1}}=2.5757$ \\
$Y$ & $\widehat{\lambda_{2}}=1.097648$ & $\widehat{v_{2}}=3.21664$ \\
\hline \hline
\end{tabular}

\subsection{Parameters Estimate $\beta_{1}, \beta_{2}$ et $\eta$}

Table 8. The coefficients of model (14)

\begin{tabular}{cccc}
\hline Parameters & $S_{\hat{\beta}}$ & $k_{j}=\frac{\hat{\beta}}{S_{\hat{\beta}}}$ & $P\left(>\left|k_{j}\right|\right)$ \\
\hline$\hat{\beta_{1}}=0.35679$ & 0.04163 & 8.57 & $<2.10^{-16} * * *$ \\
$\hat{\beta_{2}}=0.27946$ & 0.09362 & 2.985 & $0.00284 * *$ \\
$\hat{\eta}=0.27046$ & 0.19319 & 1.400 & 0.16153 \\
\hline
\end{tabular}

It is evident from the table (8), that at the level $\alpha=5 \%$ of significance, $\mathrm{p}$-value equal to 0.16153 is higher than $\alpha$; therefore the coefficient $\eta$ of estimate $\hat{\eta}=0.27046$ is null significantly; what confirm the independance between the variable $X$ and $Y$. It also evident from this table that to the same level of significance, the coefficients $\hat{\beta}_{j}(j=1,2)$ was not null significantly because the p-value was smaller than $\alpha$, what brings us to say that the factor $t$ has the effect, and on the variable $X$ and on the variable $Y$.

\section{Conclusion}

The bivariate function proposed by Kimberly et al.(2016) is not a probability distribution, so it cannot be used as a model to describe data. The bivariate COM-Poisson distribution that we have proposed in this paper is definetely a probability distribution. This distribution allowed us to highlight, not only the independence between the variables $X$ and $Y$, but also the effect of the factors (or predictors) on these variables. The resulting covariance is negative, zero or positive depending on the values of a parameter; in particular for the bivariate Poisson distribution according to Berkhout and Plug(2004).

\section{References}

Batsindila, N. P. C., Bidounga, R., \& Mizére, D. (2019). The covariance structure of the bivariate weighted Poisson distribution and application to the Aleurodicus data. Afrika Statistika, 14(2), 1999-2017. 
Berkhout, P., \& Plug, E. (2004). A bivariate Poisson count data model using conditional probabilities. Statist. Neerlandica, 58(3), 349-364. https://doi.org/10.1111/j.1467-9574.2004.00126.x

Conway, R. W., \& Maxwell, W. L. (1962). A queuing model with state dependent service rates. J. Ind. Eng., 12, 132-136.

Elion, L. L., Koukouatikissa, D. M., Bidounga, R., Louzayadio, C. G., Mizére, D., Makany, R. A., \& Kissita, G. (2016). The bivariate weighted Poisson distribution: Statistical study of the arterial hypertension data according to the weight and blood sugar level. Far East journal of Theoretical Statistics, 52(5), 365-393.

Johnson, N. L., Kotz, S., \& Balakrishnan, N. (1997). Discrete Multivariate Distribution. John Wiley and Sons Inc.

Kimberly, F. S., Darcy, S. M., \& Balakrishnan, N. (2016). Bivariate Conway-Maxwell-Poisson distribution: Formulation, properties and inference. Journal of Multivariate Analysis, 150, 152-168.

Kokonendji, C. C., Mizére, D., \& Balakrishnan, N. (2008). Connections of the Poisson weight function to overdispersion and underdispersion. Journal of Statitical Planning and inference, 138 1287-1296. https://doi.org/10.1016/j.jspi.2007.05.028

Mandangui, M. E. G. B., Bidounga, R., \& Mizére, D. (2019). Study of Aleurodicus data by the regression of the weighted Poisson distribution $Z M W D P_{1}\left(\delta_{1}, n, \pi_{0}\right)$ and $W_{P} D_{2}\left(\delta_{2}, n\right)$. Far East Journal of Mathematical Sciences, 111(2), 239-252.

Shmueli, G., Minka, T. P., Kadane, J. B., Borle, S., \& Boatwright, P. (2005). A useful distribution for fitting discrete data: revival of the Conway-Maxwell-Poisson distribution. Appl. Stat., 54(1), 127-142. https://doi.org/10.1111/j.14679876.2005.00474.x

\section{Copyrights}

Copyright for this article is retained by the author(s), with first publication rights granted to the journal.

This is an open-access article distributed under the terms and conditions of the Creative Commons Attribution license (http://creativecommons.org/licenses/by/4.0/). 